\title{
DOMINATION PROBLEM FOR NARROW ORTHOGONALLY ADDITIVE OPERATORS
}

\author{
MARAT PLIEV
}

\begin{abstract}
The "Up-and-down" theorem which describes the structure of the Boolean algebra of fragments of a linear positive operator is the well known result of the operator theory. We prove an analog of this theorem for a positive abstract Uryson operator defined on a vector lattice and taking values in a Dedekind complete vector lattice. This result we apply to prove that for an order narrow positive abstract Uryson operator $T$ from a vector lattice $E$ to a Dedekind complete vector lattice $F$, every abstract Uryson operator $S: E \rightarrow F$, such that $0 \leq S \leq T$ is also order narrow.
\end{abstract}

\section{INTRODUCTION}

Today the theory of narrow operators is an active area of Functional Analysis (see the recent monograph [23]). Lately the concept of the narrowness was generalized to the setting of orthogonally additive operators in vector lattices 21]. The aim of this article is to continue the investigation of order narrow orthogonally additive operators and to consider the domination problem for this class of operators 1

\section{PReliminaries}

The goal of this section is to introduce some basic definitions and facts. General information on vector lattices and Boolean algebras the reader can find in the books [2, 8, 9, 14.

Let $E$ be a vector lattice. A net $\left(x_{\alpha}\right)_{\alpha \in \Lambda}$ in $E$ order converges to an element $x \in E$ (notation $x_{\alpha} \stackrel{(\mathrm{o})}{\longrightarrow} x$ ) if there exists a net $\left(u_{\alpha}\right)_{\alpha \in \Lambda}$ in $E_{+}$such that $u_{\alpha} \downarrow 0$ and $\left|x_{\beta}-x\right| \leq u_{\beta}$ for all $\beta \in \Lambda$. The equality $x=\bigsqcup_{i=1}^{n} x_{i}$ means that $x=\sum_{i=1}^{n} x_{i}$ and $x_{i} \perp x_{j}$ if $i \neq j$. An element $y$ of $E$ is called a fragment (in another terminology, a component) of an element $x \in E$, provided $y \perp(x-y)$. The notation $y \sqsubseteq x$ means that $y$ is a fragment of $x$. Two fragments $x_{1}, x_{2}$

2010 Mathematics Subject Classification. Primary 47H30; Secondary 47H99.

Key words and phrases. Orthogonally additive operators, order narrow operators, fragments, vector lattices, domination problem.

1 The research was supported by Russian Foundation of Fundamental Research,the grant number 14-01-91339 
of $x$ are called mutually complemented or $M C$, in short, if $x=x_{1} \sqcup x_{2}$. If $E$ is a vector lattice and $e \in E$ then by $\mathcal{F}_{e}$ we denote the set of all fragments of $e$.

An element $e$ of a vector lattice $E$ is called a projection element if the band generated by $e$ is a projection band. A vector lattice $E$ is said to have the principal projection property if every element of $E$ is a projection element. For instance, every Dedekind $\sigma$-complete vector lattice has the principal projection property.

Definition 2.1. Let $E$ be a vector lattice, and let $F$ be a real linear space. An operator $T: E \rightarrow F$ is called orthogonally additive if $T(x+y)=T(x)+$ $T(y)$ whenever $x, y \in E$ are disjoint.

It follows from the definition that $T(0)=0$. It is immediate that the set of all orthogonally additive operators is a real vector space with respect to the natural linear operations.

Definition 2.2. Let $E$ and $F$ be vector lattices. An orthogonally additive operator $T: E \rightarrow F$ is called:

- positive if $T x \geq 0$ holds in $F$ for all $x \in E$;

- order bounded if $T$ maps order bounded sets in $E$ to order bounded sets in $F$.

An orthogonally additive, order bounded operator $T: E \rightarrow F$ is called an abstract Uryson operator. This class of operators was introduced and studied in 1990 by Mazón and Segura de León [16, 17], and then extended to lattice-normed spaces by Kusraev and the second named author [11, 12, 20]. Currently orthogonally additive operators are an active area of investigations [4, 5, 6, 21, 22,

For example, any linear operator $T \in L_{+}(E, F)$ defines a positive abstract Uryson operator by $G(f)=T|f|$ for each $f \in E$. Observe that if $T: E \rightarrow F$ is a positive orthogonally additive operator and $x \in E$ is such that $T(x) \neq 0$ then $T(-x) \neq-T(x)$, because otherwise both $T(x) \geq 0$ and $T(-x) \geq 0$ imply $T(x)=0$. So, the above notion of positivity is far from the usual positivity of a linear operator: the only linear operator which is positive in the above sense is zero. A positive orthogonally additive operator need not be order bounded. Consider, for example, the real function $T: \mathbb{R} \rightarrow \mathbb{R}$ defined by

$$
T(x)= \begin{cases}\frac{1}{x^{2}} & \text { if } x \neq 0 \\ 0 & \text { if } x=0\end{cases}
$$

The set of all abstract Uryson operators from $E$ to $F$ we denote by $\mathcal{U}(E, F)$. Consider some examples. The most famous one is the nonlinear integral Uryson operator. 
Example 2.3. Let $(A, \Sigma, \mu)$ and $(B, \Xi, \nu)$ be $\sigma$-finite complete measure spaces, and let $(A \times B, \mu \times \nu)$ denote the completion of their product measure space. Let $K: A \times B \times \mathbb{R} \rightarrow \mathbb{R}$ be a function satisfying the following condition 2 :

$\left(C_{0}\right) K(s, t, 0)=0$ for $\mu \times \nu$-almost all $(s, t) \in A \times B$;

$\left(C_{1}\right) K(\cdot, \cdot, r)$ is $\mu \times \nu$-measurable for all $r \in \mathbb{R}$;

$\left(C_{2}\right) K(s, t, \cdot)$ is continuous on $\mathbb{R}$ for $\mu \times \nu$-almost all $(s, t) \in A \times B$.

Given $f \in L_{0}(B, \Xi, \nu)$, the function $|K(s, \cdot, f(\cdot))|$ is $\nu$-measurable for $\mu$ almost all $s \in A$ and $h_{f}(s):=\int_{B}|K(s, t, f(t))| d \nu(t)$ is a well defined and $\mu$-measurable function. Since the function $h_{f}$ can be infinite on a set of positive measure, we define

$$
\operatorname{Dom}_{B}(K):=\left\{f \in L_{0}(\nu): h_{f} \in L_{0}(\mu)\right\} .
$$

Then we define an operator $T: \operatorname{Dom}_{B}(K) \rightarrow L_{0}(\mu)$ by setting

$$
(T f)(s):=\int_{B} K(s, t, f(t)) d \nu(t) \quad \mu-\text { a.e. }(\star)
$$

Let $E$ and $F$ be order ideals in $L_{0}(\nu)$ and $L_{0}(\mu)$ respectively, $K$ a function satisfying $\left(C_{0}\right)-\left(C_{2}\right)$. Then $(\star)$ defines an orthogonally additive order bounded integral operator acting from $E$ to $F$ if $E \subseteq \operatorname{Dom}_{B}(K)$ and $T(E) \subseteq F$.

Example 2.4. We consider the vector space $\mathbb{R}^{m}, m \in \mathbb{N}$ as a vector lattice with the coordinate-wise order: for any $x, y \in \mathbb{R}^{m}$ we set $x \leq y$ provided $e_{i}^{*}(x) \leq e_{i}^{*}(y)$ for all $i=1, \ldots, m$, where $\left(e_{i}^{*}\right)_{i=1}^{m}$ are the coordinate functionals on $\mathbb{R}^{m}$. Let $T: \mathbb{R}^{n} \rightarrow \mathbb{R}^{m}$. Then $T \in \mathcal{U}\left(\mathbb{R}^{n}, \mathbb{R}^{m}\right)$ if and only if there are real functions $T_{i, j}: \mathbb{R} \rightarrow \mathbb{R}, 1 \leq i \leq m, 1 \leq j \leq n$ satisfying $T_{i, j}(0)=0$ such that

$$
e_{i}^{*}\left(T\left(x_{1}, \ldots, x_{n}\right)\right)=\sum_{j=1}^{n} T_{i, j}\left(x_{j}\right),
$$

In this case we write $T=\left(T_{i, j}\right)$.

Example 2.5. Let $T: l^{2} \rightarrow \mathbb{R}$ be the operator defined by

$$
T\left(x_{1}, \ldots, x_{n}, \ldots\right)=\sum_{n \in I_{x}} n\left(\left|x_{n}\right|-1\right)
$$

where $I_{x}:=\left\{n \in \mathbb{N}:\left|x_{n}\right| \geq 1\right\}$. It is not difficult to check that $T$ is a positive abstract Uryson operator.

Example 2.6. Let $(\Omega, \Sigma, \mu)$ be a measure space, $E$ a sublattice of the vector lattice $L_{0}(\mu)$ of all equivalence classes of $\Sigma$-measurable functions $x: \Omega \rightarrow \mathbb{R}$, $F$ a vector lattice and $\nu: \Sigma \rightarrow F$ a finitely additive measure. Then the map $T: E \rightarrow F$ given by $T(x)=\nu(\operatorname{supp} x)$ for any $x \in E$, is an abstract Uryson operator which is positive if and only if $\nu$ is positive.

Consider the following order in $\mathcal{U}(E, F): S \leq T$ whenever $T-S$ is a positive operator. Then $\mathcal{U}(E, F)$ becomes an ordered vector space. If a vector lattice $F$ is Dedekind complete we have the following theorem.

\footnotetext{
${ }^{2}\left(C_{1}\right)$ and $\left(C_{2}\right)$ are called the Carathéodory conditions
} 
Theorem 2.7. (16], Theorem 3.2). Let $E$ and $F$ be a vector lattices, $F$ Dedekind complete. Then $\mathcal{U}(E, F)$ is a Dedekind complete vector lattice. Moreover for $S, T \in \mathcal{U}(E, F)$ and for $f \in E$ following hold

(1) $(T \vee S)(f):=\sup \left\{T g_{1}+S g_{2}: f=g_{1} \sqcup g_{2}\right\}$.

(2) $(T \wedge S)(f):=\inf \left\{T g_{1}+S g_{2}: f=g_{1} \sqcup g_{2}\right\}$.

(3) $(T)^{+}(f):=\sup \{T g: g \sqsubseteq f\}$.

(4) $(T)^{-}(f):=-\inf \{T g: g ; g \sqsubseteq f\}$.

(5) $|T f| \leq|T|(f)$.

We follow 21] in the next definition.

Definition 2.8. Let $E, F$ be vector lattices with $E$ an atomless. An abstract Uryson operator $T: E \rightarrow F$ is called order narrow if for every $e \in E$ there exists a net of decompositions $e=f_{\alpha} \sqcup g_{\alpha}$ such that $\left(T\left(f_{\alpha}\right)-T\left(g_{\alpha}\right)\right) \stackrel{(\mathrm{o})}{\longrightarrow} 0$.

It is a worth noting that linear order narrow operators were firstly introduced by Maslyuchenko, Mykhaylyuk and Popov in [15. Lately, in setting of lattice-normed spaces linear order narrow operators were investigated by the author in [19].

Acknowledgment. Author is very grateful to Mikhail Popov for the valuable remarks and useful discussions.

\section{The Boolean algebra of fragments of a Positive Uryson} OPERATOR

Let $E, F$ be vector lattices with $F$ Dedekind complete and $T \in \mathcal{U}_{+}(E, F)$. The purpose of this section is to describe the fragments of $T$. That is

$$
\mathcal{F}_{T}=\left\{S \in \mathcal{U}_{+}(E, F): S \wedge(T-S)=0\right\} .
$$

Like in the linear case we consider elementary fragments. For a subset $\mathcal{A}$ of a vector lattice $W$ we employ the following notation:

$$
\begin{gathered}
\mathcal{A}^{1}=\left\{x \in W: \exists \text { a sequence }\left(x_{n}\right) \subset \mathcal{A} \text { with } x_{n} \uparrow x\right\} ; \\
\mathcal{A}^{\uparrow}=\left\{x \in W: \exists \text { a net }\left(x_{\alpha}\right) \subset \mathcal{A} \text { with } x_{\alpha} \uparrow x\right\} .
\end{gathered}
$$

The meanings of $\mathcal{A}^{\downarrow}$ and $\mathcal{A}^{\downarrow}$ are analogous. As usual, we also write

$$
\mathcal{A}^{\downarrow \uparrow}=\left(\mathcal{A}^{\downarrow}\right)^{\uparrow} ; \mathcal{A}^{1 \downarrow \uparrow}=\left(\left(\mathcal{A}^{1}\right)^{\downarrow}\right)^{\uparrow} \text {. }
$$

It is clear that $\mathcal{A}^{\downarrow \downarrow}=\mathcal{A}^{\downarrow}, \mathcal{A}^{\uparrow \uparrow}=\mathcal{A}^{\uparrow}$. Consider a positive abstract Uryson operator $T: E \rightarrow F$, where $F$ is Dedekind complete. Since $\mathcal{F}_{T}$ is a Boolean algebra, it is closed under finite suprema and infima. In particular, all "ups and downs" of $\mathcal{F}_{T}$ are likewise closed under finite suprema and infima, and therefore they are also directed upward and, respectively, downward.

Definition 3.1. A subset $D$ of a vector lattice $E$ is called a lateral ideal if the following conditions hold

(1) if $x \in D$ then $y \in D$ for every $y \in \mathcal{F}_{x}$;

(2) if $x, y \in D, x \perp y$ then $x+y \in D$. 
Consider some examples.

Example 3.2. Let $E$ be a vector lattice. Every order ideal in $E$ is a lateral ideal.

Example 3.3. Let $E, F$ be a vector lattices and $T \in \mathcal{U}_{+}(E, F)$. Then $\mathcal{N}_{T}:=$ $\{e \in E: T(e)=0\}$ is a lateral ideal.

The following example is important for further considerations.

Lemma 3.4. (4, Lemma 3.5). Let $E$ be a vector lattice and $x \in E$. Then $\mathcal{F}_{x}$ is a lateral ideal.

Let $T \in \mathcal{U}_{+}(E, F)$ and $D \subset E$ be a lateral ideal. Then for every $x \in E$, we define a map $\pi^{D} T: E \rightarrow F_{+}$by the following formula

$$
\pi^{D} T(x)=\sup \left\{T y: y \in \mathcal{F}_{x} \cap D\right\} .
$$

Lemma 3.5. (4, Lemma 3.6). Let $E, F$ be vector lattices with $F$ Dedekind complete, $\rho \in \mathfrak{B}(F), T \in \mathcal{U}_{+}(E, F)$ and $D$ be a lateral ideal. Then $\pi^{D} T$ is a positive abstract Uryson operator and $\rho \pi^{D} T \in \mathcal{F}_{T}$.

If $D=\mathcal{F}_{x}$ then the operator $\pi^{D} T$ is denoted by $\pi^{x} T$. Let $F$ be a vector lattice. Recall that a family of mutually disjoint order projections $\left(\rho_{\xi}\right)_{\xi \in \Xi}$ on $F$ is said to be partition of unity if $\bigvee_{\xi \in \Xi}\left(\rho_{\xi}\right)_{\xi \in \Xi}=I d_{F}$. Any fragment of the form $\sum_{i=1}^{n} \rho_{i} \pi^{x_{i}} T, n \in \mathbb{N}$, where $\rho_{1}, \ldots, \rho_{n}$ is a finite family of mutually disjoint order projections in $F$, like in the linear case is called an elementary fragment $T$. The set of all elementary fragments of $T$ we denote by $\mathcal{A}_{T}$.

For further considerations we need the following auxiliary proposition, which was proven by nonstandard methods.

Lemma 3.6 ([10], Proposition 5.2.7.2). Let $F$ be a Dedekind complete vector lattice with a weak order unit $u$ and $\left(x_{\lambda}\right)_{\lambda \in \Lambda}$ be an order bounded net in $F$. Then the net $\left(x_{\lambda}\right)_{\lambda \in \Lambda}$ order converges to an element $x \in F$ if and only if for every $\varepsilon>0$ there exists a partition of unity $\left(\rho_{\lambda}\right)_{\lambda \in \Lambda}$ such that

$$
\rho_{\lambda}\left|x_{\beta}-x\right| \leq \varepsilon u, \quad \beta \geq \lambda .
$$

Remark 3.7. Observe that every Dedekind complete vector lattice is an order dense ideal in some Dedekind complete vector lattice with a weak order unit (24, Theorem 4.7.2).

Lemma 3.8. Let $E, F$ be vector lattices, $F$ be Dedekind complete and let $\mathfrak{A}$ be the set of all weak order units in $F$. If operators $T, S \in \mathcal{U}_{+}(E, F)$ are disjoint, then for every $x \in E, u \in \mathfrak{A}$ and $\varepsilon>0$ there exists a partition of unity $\left(\pi_{\xi}\right)_{\xi \in \Xi}$ in $\mathfrak{B}(F)$ and a family $\left(x_{\xi}\right)_{\xi \in \Xi}$ of fragments of $x$, such that

$$
\pi_{\xi}\left(T x_{\xi}+S\left(x-x_{\xi}\right)\right) \leq \varepsilon u \text { for all } \xi \in \Xi .
$$

\footnotetext{
${ }^{3}$ An element $u \in F_{+}$is a weak order unit if $\{u\}^{\perp \perp}=F$, i.e. except 0 there are no elements in $F$ which are disjoint to $u$.
} 
Proof. Take any $x \in E$. Denote by $\Xi$, the set of all pairs $\xi=(y, z) \in$ $\mathcal{F}_{x} \times \mathcal{F}_{x}$ of mutually disjoint fragments of $x$, such that $y+z=x$. For any $\xi=(y, x-y) \in \Xi$ put $f_{\xi}=T y+S(x-y)$. Due to formula (2) of Theorem 2.7 the disjointness of the operators $S$ and $T$ implies $\inf _{\xi \in \Xi}\left\{f_{\xi}\right\}=0$. Denote by $\Delta$ the collection of all finite subsets of $\Xi$ ordered as usual by inclusion, i.e. $\alpha \leq \alpha^{\prime}$ iff $\alpha \subset \alpha^{\prime}$. Introduce a set $\left(y_{\alpha}\right)_{\alpha \in \Delta}$ of all infima of finitely many elements of the set $\left\{f_{\xi}: \xi \in \Xi\right\}$, i.e. if $\alpha \in \Delta$ is a finite set $\alpha=\left\{\xi_{\alpha_{1}}, \ldots, \xi_{\alpha_{n}}\right\}$, where $\xi_{\alpha_{k}} \in \Xi$ for $k=1, \ldots, n$, then

$$
y_{\alpha}=\bigwedge_{i=1}^{n} f_{\xi_{\alpha_{i}}}
$$

The set $\left(y_{\alpha}\right)_{\alpha \in \Delta}$ is downwards directed and $o-\lim _{\alpha \in \Delta} y_{\alpha}=0$. By Proposition 3.6. for every $\varepsilon>0$ and $u \in \mathfrak{A}$ there exists a partition of unity $\left(\rho_{\alpha}\right)_{\alpha \in \Delta}$ in $\mathfrak{B}(F)$ such that

$$
\rho_{\alpha}\left(y_{\alpha}\right) \leq \varepsilon u \text { for all } \alpha \in \Delta .
$$

In particular, $\rho_{\alpha}\left(f_{\xi}\right)<\varepsilon u$ if $\alpha=\xi$.

Identify now $F$ with a vector sublattice of the Dedekind complete vector lattice $C_{\infty}(Q)$ of all extended real valued continuous functions on some extremally disconnected compact space $Q$ (more exactly with its image under some vector lattice isomorphism), where the choosen weak order unit $u$ is mapped onto the constant function 1 on $Q$ (see [1, Theorem 3.35). Then the order projections $\left(\rho_{\alpha}\right)_{\alpha \in \Delta}$ (of the above partition of unity) are the multiplication operators in the space $C_{\infty}(Q)$ generated by the characteristic functions $\mathbf{1}_{Q_{\alpha}}$, respectively, where $Q_{\alpha}$ for all $\alpha \in \Delta$ are closed-open subsets of $Q$ such that $Q=\bigcup_{\alpha} Q_{\alpha}$ and $Q_{\alpha} \cap Q_{\alpha^{\prime}}=\emptyset$ for every $\alpha, \alpha^{\prime} \in \Delta, \alpha \neq \alpha^{\prime}$. The supremum $\sup \rho_{\alpha}$ is the identity operator $I_{F}$.

$$
\alpha \in \Delta
$$

For $\alpha \in \Delta$ and $\xi \in \Xi$ define the set

$$
A_{\xi}^{\alpha}=\left\{t \in Q_{\alpha}: f_{\xi}(t)<f_{\beta}(t), \beta \in \alpha, \beta \neq \xi\right\}
$$

and denote by $\overline{A_{\xi}^{\alpha}}$ its closure in $Q_{\alpha}$ and, consequently in $Q$. So $\overline{A_{\xi}^{\alpha}}$ are closed-open subsets of $Q$ for every $\alpha \in \Delta, \xi \in \Xi$ and, mutually disjoint if at least one index is different $\xi \neq \xi^{\prime}$ or $\alpha \neq \alpha^{\prime}$. Denote by $\rho_{\xi}^{\alpha}$ the multiplication operator generated by the characteristic function $\mathbf{1}_{\overline{A_{\xi}^{\alpha}}}$, i.e. $\rho_{\xi}^{\alpha}(f)=f \cdot \mathbf{1}_{\overline{A_{\xi}^{\alpha}}}$ for any function $f \in C_{\infty}(Q)$. It is clear that $\rho_{\xi}^{\alpha}$ is an order projection in $C_{\infty}(Q)$ and $\overline{A_{\xi}^{\alpha}} \subset Q_{\alpha}$ implies $\rho_{\xi}^{\alpha} \leq \rho_{\alpha}$. Hence $\rho_{\xi}^{\alpha}\left(f_{\xi}\right) \leq \varepsilon u$ for every $\xi \in \Xi$ and every $\alpha \in \Delta$. By what has been mentioned above the order projections $\rho_{\xi}^{\alpha}$ are mutually disjoint, whenever $\xi \neq \xi^{\prime}$ or $\alpha \neq \alpha^{\prime}$. Therefore, the order projections $\pi_{\xi}=\sup _{\alpha \in \Delta} \rho_{\xi}^{\alpha}$ and $\pi_{\xi^{\prime}}=\sup _{\alpha \in \Delta} \rho_{\xi^{\prime}}^{\alpha}$ are mutually disjoint as well. We show that the supremum of all $\pi_{\xi}$ is the identity operator. By assuming the contrary there is a nonzero order projection $\gamma$ which is disjoint to each projection $\pi_{\xi}$ what causes its disjointness to each $\rho_{\xi}^{\alpha}$ and finally, $\gamma$ is disjoint 
to each $\rho_{\alpha}$. This contradicts the fact that $\left(\rho_{\alpha}\right)_{\alpha \in \Delta}$ is a partition of unity. Thus $\left(\pi_{\xi}\right)_{\xi \in \Xi}$ is a partition of unity and

$$
\pi_{\xi}\left(T x_{\xi}+S\left(x-x_{\xi}\right)\right) \leq \varepsilon u \text { for every } \xi \in \Xi .
$$

Lemma 3.9. Let $E, F, \mathfrak{A}_{F}$ be the same as in the Lemma 3.8, $S, T \in \mathcal{U}_{+}(E, F)$. If $S \perp T$, then for every $x \in E, \varepsilon>0, \mathbf{1} \in \mathfrak{A}_{F}$ there exists a partition of unity $\left(\rho_{\xi}\right)_{\xi \in \Xi}$ in $\mathfrak{B}(F)$, and a family $\left(x_{\xi}\right)_{\xi \in \Xi}$ of fragments of $x$ such that $\rho_{\xi} \pi^{x_{\xi}} T(x) \leq \varepsilon \mathbf{1}$ and $\rho_{\xi}\left(S-\rho_{\xi} \pi^{x_{\xi}} S\right) x \leq \varepsilon \mathbf{1}$ for every $\xi \in \Xi$.

Proof. Observe that for every $y \in \mathcal{F}_{x}, x \in E$ we have $\pi^{y} T x=T y$. Fix a weak order unit 1 and $\varepsilon>0$. By Lemma 3.8 there exist a partition of unity $\left(\rho_{\xi}\right)_{\xi \in \Xi}$ in $F$, and a family $\left(x_{\xi}\right)_{\xi \in \Xi}$ of fragments of $x$ such that

$$
\rho_{\xi}\left(T x_{\xi}+S\left(x-x_{\xi}\right)\right) \leq \varepsilon u \text { for all } \xi \in \Xi \text {. }
$$

Consequently, $\rho_{\xi} T x_{\xi}=\rho_{\xi} \pi^{x_{\xi}} T x \leq \varepsilon \mathbf{1}$ and

$$
\rho_{\xi} S\left(x-x_{\xi}\right)=\rho_{\xi} S x-\rho_{\xi} S x_{\xi}=\rho_{\xi}\left(S-\rho_{\xi} \pi^{x_{\xi}} S\right) x \leq \varepsilon \mathbf{1} .
$$

Lemma 3.10. Let $E, F, \mathfrak{A}_{F}$ be the same as in the Lemma 3.8, $T \in \mathcal{U}_{+}(E, F)$. If $S \in \mathcal{F}_{T}$ then for every $x \in E, \varepsilon>0, \mathbf{1} \in \mathfrak{A}_{F}$ there exists a partition of unity $\left(\rho_{\xi}\right)_{\xi \in \Xi}$ in $\mathfrak{B}(F)$, and a family $\left(x_{\xi}\right)_{\xi \in \Xi}$ of fragments of $x$, such that $\rho_{\xi}\left|S-\rho_{\xi} \pi^{x_{\xi}} T\right| x \leq \varepsilon \mathbf{1}$ for every $\xi \in \Xi$.

Proof. Using Lemma 3.9 we have

$$
\begin{gathered}
\rho_{\xi}\left|S-\rho_{\xi} \pi^{x_{\xi}} T\right| x \leq \rho_{\xi}\left|S-\rho_{\xi} \pi^{x_{\xi}} S\right| x+\rho_{\xi}\left|\rho_{\xi} \pi^{x_{\xi}} S-\rho_{\xi} \pi^{x_{\xi}} T\right| x= \\
=\rho_{\xi}\left|S-\rho_{\xi} \pi^{x_{\xi}} S\right| x+\rho_{\xi}\left|\rho_{\xi} \pi^{x_{\xi}}(T-S)\right| x \leq \varepsilon \mathbf{1} .
\end{gathered}
$$

Lemma 3.11. Let $E, F$ be the same as in Lemma [3.9, $T \in \mathcal{U}_{+}(E, F)$ and $S \in \mathcal{F}_{T}$. Then

(1) for every $x \in E, \varepsilon>0, \mathbf{1} \in \mathfrak{A}_{F}$ there exists $G_{x} \in \mathcal{A}_{T}^{\uparrow}$, so that $\left|S-G_{x}\right| x \leq \varepsilon \mathbf{1}$

(2) for every $x \in E$ there exists $R_{x} \in \mathcal{A}_{T}^{\uparrow\rfloor}$, so that $\left|S-R_{x}\right| x=0$.

Proof. Let us to prove (1). By Lemma 3.10 there exists a partition of unity $\left(\rho_{\xi}\right)_{\xi \in \Xi}$ in $\mathfrak{B}(F)$, and a family $\left(x_{\xi}\right)_{\xi \in \Xi}$ of fragments of $x$ such that $\rho_{\xi} \mid S-$ $\rho_{\xi} \pi^{x_{\xi}} T \mid x \leq \varepsilon \mathbf{1}$ for $\xi \in \Xi$. By $\Delta$ we denote the system of all finite subsets of $\Xi$. It is ordered by inclusion. Surely, $\Delta$ is a directed set. For every $\theta \in \Delta$ set $G_{\theta}=\sum_{\theta \in \Delta} \rho_{\xi} \pi^{x_{\xi}} T$. The net $\left(G_{\theta}\right)_{\theta \in \Delta}$ is increasing. Let $G_{x}=\sup \left(G_{\theta}\right)_{\theta \in \Delta}$.

Then $G_{x} \in \mathcal{A}_{T}^{\uparrow}$ and we may write

$$
\rho_{\xi}\left|S-G_{\theta}\right| x=\rho_{\xi}\left|S-\sum_{\theta \in \Delta} \rho_{\xi} \pi^{x_{\xi}} T\right| x \leq \varepsilon \mathbf{1}
$$


for every $\xi \in \Xi$ and every $\theta \geq\{\xi\}$. Therefore $\rho_{\xi}\left|S-G_{x}\right| x \leq \varepsilon \mathbf{1}$ for every $\xi \in \Xi$ and $\left|S-G_{x}\right| x \leq \varepsilon \mathbf{1}$.

Now we prove (2). Fix any $\mathbf{1} \in \mathfrak{A}_{F}$. For $\varepsilon_{n}=\frac{1}{2^{n}}$ there exists $G_{x}^{n} \in \mathcal{A}_{T}$ such that $\left|S-G_{x}^{n}\right| x \leq \frac{1}{2^{n}} \mathbf{1}$. Let $C_{x}^{k}=\bigvee_{n=k}^{\infty} G_{x}^{n}$ and $C_{x}^{k, i}=\bigvee_{n=k}^{n=k+i} G_{x}^{n}$. Since $\mathcal{A}_{T}$ is a subalgebra of $\mathcal{F}_{T}$, one has $C_{x}^{k, i} \in \mathcal{A}_{T}^{\uparrow}$ and $C_{x}^{k, i} \uparrow C_{x}^{k} \in \mathcal{A}_{T}^{\uparrow 1}=\mathcal{A}_{T}^{\uparrow}$. Then we have

$$
\begin{gathered}
\left|S-C_{x}^{k, i}\right| x=\left|S-\bigvee_{n=k}^{n=k+i} G_{x}^{n}\right| x=\left|\bigwedge_{n=k}^{n=k+i}\left(S-G_{x}^{n}\right)\right| x \leq \\
\leq \sum_{n=k}^{n=k+i}\left|S-G_{x}^{n}\right| x \leq \sum_{n=k}^{\infty} \frac{1}{2^{n}} \mathbf{1} \leq \frac{1}{2^{k-1}} \mathbf{1}
\end{gathered}
$$

So we may write $\left|S-C_{x}^{k}\right| \leq \frac{1}{2^{k-1}} \mathbf{1}$. The sequence $\left(C_{x}^{k}\right)$ is decreasing. Let $R_{x}=\inf C_{x}^{k}$. Then $R_{x} \in \mathcal{A}_{T}^{\uparrow \downarrow}$ and $\left|S-R_{x}\right| x=0$.

Remark 3.12. Observe that $R_{x} y=0$ for every $y$ such that $\mathcal{F}_{x} \cap \mathcal{F}_{y}=0$. Moreover, if $y \in \mathcal{F}_{x}$ and $\left|S-R_{x}\right| x=0$ we can write $0 \leq\left|S-R_{x}\right| y \leq$ $\left|S-R_{x}\right| x=0$, and therefore $\left|S-R_{x}\right| y=0$ for every $y \in \mathcal{F}_{x}$.

Lemma 3.13. Let $E, F$ be the same as in Lemma [3.11, $T \in \mathcal{U}_{+}(E, F)$, $x \in E$ and $S \in \mathcal{F}_{T}$. Then there exists $a G \in \mathcal{A}_{T}^{\uparrow \downarrow}$ such that:

$$
0 \leq G \leq S \text { and } G x=S x .
$$

Proof. Fix $x \in E$ and let

$$
W:=\left\{R \in \mathcal{A}_{T}^{\uparrow \uparrow}:|S-R| x=0\right\} .
$$

By Lemma 3.11 the set $W$ is nonempty, and an easy argument shows that $W$ is directed downward. Let $G=\inf \{W\}$. Clearly, $G \in \mathcal{A}_{T}^{\uparrow \downarrow \downarrow}=\mathcal{A}_{T}^{\uparrow \downarrow}$, and hence $|S-G| x=0$ We claim that $0 \leq G \leq S$. By Remark $3.12 G z=0$ for every $z \in E$, such that $\mathcal{F}_{z} \cap \mathcal{F}_{x}=0$ and we must prove $(G-S)^{+} y=0$ for every $y \in \mathcal{F}_{x}$. Now we may write

$$
(G-S)^{+} y \leq\left|R_{x}-S\right| y=\left|S-R_{x}\right| y=0,
$$

where $y \in \mathcal{F}_{x}$ and $R_{x}$ is a some element of $W$.

The following theorem is the first main result of the article.

Theorem 3.14. Let $E, F$ be vector lattices, $F$ Dedekind complete, $T \in$ $\mathcal{U}_{+}(E, F)$ and $S \in \mathcal{F}_{T}$. Then $S \in \mathcal{A}_{T}^{\uparrow \downarrow \uparrow}$.

Proof. Let $S \in \mathcal{F}_{T}$ be fixed, and let

$$
W=\left\{R \in \mathcal{A}_{T}^{\uparrow \downarrow}: 0 \leq R \leq S\right\}
$$

Clearly, $W$ is a directed set, and by Lemma 3.13 we know that $W \neq \emptyset$. Let $G=\sup \{W\}$, and remark that $0 \leq G \leq S$. On the other hand, if $x \in E$ is an arbitrary element of $E$, by Lemma 3.13 there exists some $R \in W$, such 
that $0 \leq R \leq G \leq S$ and $R x=S x$. Thus $G=S, S \in \mathcal{A}_{T}^{\uparrow \downarrow \uparrow}$ and $\mathcal{F}_{T}=\mathcal{A}_{T}^{\uparrow \uparrow \uparrow}$.

Remark that for linear positive operators the same theorem and its modifications were proved by de Pagter, Aliprantis and Burkinshaw, Kusraev and Strizhevski in [3, 13, 18].

\section{Domination problem For abstract Uryson narrow operators}

In this section we consider a domination problem for narrow abstract Uryson operators. In the classical sense, the domination problem can be stated as follows. Let $E, F$ be vector lattices, $S, T: E \rightarrow F$ linear operators with $0 \leq S \leq T$. Let $\mathcal{P}$ be some property of linear operators $R: E \rightarrow F$, so that $\mathcal{P}(R)$ means that $R$ possesses $\mathcal{P}$. Does $\mathcal{P}(T)$ imply $\mathcal{P}(S)$ ?

Let $E$ be a vector lattice and $x \in E_{+}$. The order ideal generated by $x$ we denote by $E_{x}$. The following theorem is an important tool for further considerations.

Theorem 4.1. (Freudenthal Spectral Theorem)(2], Theorem 2.8). Let E be a vector lattice with the principal projection property and let $x \in E_{+}$. Then for every $y \in E_{x}$ there exists a sequence $\left(u_{n}\right)$ of $x$-step functions satisfying $0 \leq y-u_{n} \leq \frac{1}{n} x$ for each $n$ and $u_{n} \uparrow y$.

The next theorem is the second main result of the article.

Theorem 4.2. Let $E, F$ be vector lattices, $E$ atomless and with the principal projection property, $F$ be Dedekind complete, and $T \in \mathcal{U}_{+}(E, F)$ be an order narrow operator. Then every operator $S \in \mathcal{U}_{+}(E, F)$, such that $0 \leq S \leq T$ is order narrow as well.

For the proof we need an some auxiliary result. Let $E, F$ be vector lattices, a family of operators $\left\{T_{1}, \ldots, T_{n}\right\} \subset \mathcal{U}(E, F)$ is said to have pairwise disjoint supports if there exists a family of pairwise disjoint bands $E_{1}, \ldots, E_{n} \subset E$, such that $T_{i} x=0$ for every $x \in E_{i}^{\perp}, i \in\{1, \ldots, n\}$.

Lemma 4.3. Let $E, F$ be vector lattices, $E$ atomless and with the projection property, $F$ be Dedekind complete, and $\left\{T_{1}, \ldots, T_{n}\right\} \subset \mathcal{U}(E, F)$ be a family of order narrow operators with pairwise disjoint supports. Then $T=\sum_{i=1}^{n} T_{i}$ is an order narrow operator as well.

Proof. Fix an arbitrary element $e \in E$. Let $\rho_{i}$, be a band projection to the band $E_{i}, i \in\{1, \ldots, n\}$, and $\zeta=I d-\bigvee_{i=1}^{n} \rho_{i}$. Then we may write $e=h \sqcup \bigsqcup_{i=1}^{n} e_{i}$, where $e_{i}=\rho_{i} e, i \in\{1, \ldots, n\}$ and $h=\zeta e$. By our assumption for every $e_{i}$, $i \in\{1, \ldots, n\}$ there exists a net of decompositions $e_{i}=e_{i 1}^{\alpha} \sqcup e_{i 2}^{\alpha}$ such that 


$$
\begin{gathered}
\left(T_{i}\left(e_{i 1}^{\alpha}\right)-T_{i}\left(e_{i 2}^{\alpha}\right)\right) \stackrel{(\mathrm{o})}{\longrightarrow} 0 . \text { Let } f_{\alpha}=\bigsqcup_{i=1}^{n} e_{i 1}^{\alpha} \text { and } g_{\alpha}=\bigsqcup_{i=1}^{n} e_{i 2}^{\alpha} \text {. Now we have } \\
\qquad\left|T\left(h+f_{\alpha}\right)-T\left(g_{\alpha}\right)\right|=\left|\sum_{i=1}^{n} T_{i}\left(h \sqcup \bigsqcup_{j=1}^{n} e_{j 1}^{\alpha}\right)-\sum_{i=1}^{n} T_{i}\left(\bigsqcup_{j=1}^{n} e_{j 2}^{\alpha}\right)\right|= \\
\left|\sum_{i=1}^{n} T_{i}\left(e_{i 1}^{\alpha}\right)-\sum_{i=1}^{n} T_{i}\left(e_{i 2}^{\alpha}\right)\right|=\mid \sum_{i=1}^{n}\left(T_{i}\left(e_{i 1}^{\alpha}\right)-T_{i}\left(e_{i 2}^{\alpha}\right) \mid \leq\right. \\
\sum_{i=1}^{n}\left|T_{i}\left(e_{i 1}^{\alpha}\right)-T_{i}\left(e_{i 2}^{\alpha}\right)\right| \stackrel{(\mathrm{o})}{\longrightarrow} 0 .
\end{gathered}
$$

Thus $\left(h \sqcup f_{\alpha}\right) \sqcup g_{\alpha}=e$ is the desired net of decompositions.

Lemma 4.4. Let $E, F$ be the same as in the Theorem 4.2, $x_{1}, x_{2} \in E$ and $x_{1} \perp x_{2}$. Then $\pi^{x_{1}+x_{2}} T=\pi^{x_{1}} T+\pi^{x_{2}} T$ for every $T \in \mathcal{U}_{+}(E, F)$.

Proof. Fix an arbitrary element $x \in E$. Then for every $y \in \mathcal{F}_{x}$ so that $y \sqsubseteq\left(x_{1}+x_{2}\right)$, we have $y=y_{1} \sqcup y_{2}, y_{i} \sqsubseteq x_{i}, i \in\{1,2\}$ and the following inequalities hold

$$
\begin{aligned}
T y=T y_{1}+T y_{2} & \leq \pi^{x_{1}} T x+\pi^{x_{2}} T x \\
\pi^{x_{1}+x_{2}} T x & \leq \pi^{x_{1}} T x+\pi^{x_{2}} T x .
\end{aligned}
$$

On the other hand for every $y_{i} \sqsubseteq x_{i}, y_{i} \sqsubseteq x, i \in\{1,2\}$ we may write

$$
\begin{aligned}
T y_{1}+T y_{2}=T\left(y_{1}+y_{2}\right) & \leq \pi^{x_{1}+x_{2}} T x ; \\
\pi^{x_{1}} T x+\pi^{x_{2}} T x & \leq \pi^{x_{1}+x_{2}} T x .
\end{aligned}
$$

Proof of Theorem 4.2. Let $T \in \mathcal{U}_{+}(E, F)$ be an order narrow operator, and $x \in E$. Firstly we prove that operator $\rho \pi^{x} T$ is also order narrow. Fix an arbitrary element $e \in E$. By our assumption there exists a net of decompositions $e=f_{\alpha} \sqcup g_{\alpha}$ such that $\left|T\left(f_{\alpha}\right)-T\left(g_{\alpha}\right)\right| \leq \eta_{\alpha},\left(\eta_{\alpha}\right) \subset F_{+}$and $\left(\eta_{\alpha}\right) \downarrow 0$. Remark that $D=\left\{y \sqsubseteq e: y \in \mathcal{F}_{x}\right\}$ is a directed set and by definition of the operator $\pi^{x} T$ there exists a net $\left(y_{\alpha}\right) \subset D$ so that

$$
\left|\pi^{x} T e-T y_{\alpha}\right|=\left|\pi^{x} T e-\pi^{x} T y_{\alpha}\right|=\left|\pi^{x} T\left(e-y_{\alpha}\right)\right| \leq \xi_{\alpha}
$$

for some decreasing net $\left(\xi_{\alpha}\right) \subset F_{+}, \inf _{\alpha} \xi_{\alpha}=0$. By our assumption there exists a net of decompositions $y_{\alpha}=f_{\alpha} \sqcup g_{\alpha}$ such that $\left|T\left(f_{\alpha}\right)-T\left(g_{\alpha}\right)\right| \leq \eta_{\alpha}$, $\left(\eta_{\alpha}\right) \subset F_{+}$and $\left(\eta_{\alpha}\right) \downarrow 0$. Then we may write

$$
\begin{array}{r}
\left.\mid \pi^{x} T\left(\left(e-y_{\alpha}\right) \sqcup f_{\alpha}\right)\right)-\pi^{x} T\left(g_{\alpha}\right) \mid= \\
\left|\pi^{x} T\left(e-y_{\alpha}\right)+\pi^{x} T f_{\alpha}-\pi^{x} T g_{\alpha}\right|= \\
\left|\pi^{x} T\left(e-y_{\alpha}\right)+T f_{\alpha}-T g_{\alpha}\right| \leq \\
\left|\pi^{x} T\left(e-y_{\alpha}\right)\right|+\left|T f_{\alpha}-T g_{\alpha}\right| \leq \xi_{\alpha}+\eta_{\alpha} \stackrel{(\mathrm{o})}{\longrightarrow} 0 .
\end{array}
$$


So $\left.\left(\left(e-y_{\alpha}\right) \sqcup f_{\alpha}\right)\right) \sqcup g_{\alpha}=e$ is a desired net of decompositions. It is clear that operator $\rho \pi^{x} T$ is order narrow as well. Secondly, take the operator $R=\sum_{i=1}^{n} \rho_{i} \pi^{x_{i}} T$, where $x_{1}, \ldots, x_{n}$ are fragments of a some element $x \in E$ and $\rho_{1}, \ldots, \rho_{n}$ are mutually disjoint. By Lemma 4.4, we may assume that all fragments $x_{1}, \ldots, x_{n}$ are mutually disjoint. Then applying Lemma 4.3 we prove that $R$ is an order narrow operator. Now, let $\left(R_{\xi}\right)_{\xi \in \Xi} \subset \mathcal{U}_{+}(E, F)$ be an increasing (decreasing) net of order narrow operators and $S=\sup R_{\xi}$ $\left(S=\inf _{\xi} R_{\xi}\right)$. This meant that there exists a decreasing net $\left(G_{\xi}\right)_{\xi \in \Xi \subset}^{\xi} \subset$ $\mathcal{U}_{+}(E, F)$, so that $\inf _{\xi} G_{\xi}=0$ and

$$
\left|S e-R_{\xi} e\right|=\left|\left(S-R_{\xi}\right) e\right| \leq\left|S-R_{\xi}\right| e \leq G_{\xi} e
$$

for every $e \in E$. Let us show that $S$ is also order narrow. Indeed, fix an arbitrary element $e \in E$ and write

$$
\begin{array}{r}
\left|S f_{\alpha}-S g_{\alpha}\right|=\left|S f_{\alpha}-R_{\xi} f_{\alpha}+R_{\xi} f_{\alpha}-R_{\xi} g_{\alpha}+R_{\xi} g_{\alpha}-S g_{\alpha}\right| \leq \\
\left|S f_{\alpha}-R_{\xi} f_{\alpha}\right|+\left|R_{\xi} f_{\alpha}-R_{\xi} g_{\alpha}\right|+\left|S g_{\alpha}-R_{\xi} g_{\alpha}\right| \leq \\
G_{\xi} f_{\alpha}+\left|R_{\xi} f_{\alpha}-R_{\xi} g_{\alpha}\right|+G_{\xi} g_{\alpha} \leq \\
G_{\xi} e+\left|R_{\xi} f_{\alpha}-R_{\xi} g_{\alpha}\right|+G_{\xi} \stackrel{\text { (o) }}{\longrightarrow} 0 .
\end{array}
$$

By Theorem 3.14 we have that $\mathcal{F}_{T}=\mathfrak{A}_{T}^{\uparrow \uparrow \uparrow}$ and applying this equality we obtain that every fragment of an order narrow operator $T$ is also order narrow. Finally take an arbitrary operator $S \in \mathcal{U}(E, F)$, so that $0 \leq S \leq k T$, $k \in \mathbb{R}_{+}$. By Theorem 4.1 there exists a sequence $R_{n}$ of $T$-step positive abstract Uryson operators $R_{n}=\sum_{i=1}^{k_{n}} \lambda_{i} C_{i}$, where $\lambda_{i}>0$ for $i \in\{1, \ldots, n\}$ and the operators $C_{1}, \ldots, C_{k_{n}}$ are pairwise disjoint fragments of $T$ such that so that $\left|S(e)-R_{n}(e)\right| \leq \frac{1}{n} T(e)$ for every $e \in E$. Dividing by $\max \left\{\lambda_{i}: i=\right.$ $\left.1, \ldots, k_{n}\right\}$ we may assume that $\lambda_{i} \leq 1$ for every $i \in\left\{1, \ldots, k_{n}\right\}$ and therefore $0 \leq R_{n}=\sum_{i=1}^{k_{n}} \lambda_{i} C_{i}=\bigvee_{i=1}^{k_{n}} \lambda_{i} C_{i} \leq T$ is a fragment of the operator $T$ for every $n \in \mathbb{N}$. Thus $R_{n}$ is an order narrow operator for every $n \in \mathbb{N}$. Finally, using the same arguments as above, we obtain that $S$ is an order narrow operator.

Remark that for linear positive operators the similar theorem was proved by Flores and Ruiz in [7].

\section{REFERENCES}

[1] Yu. A. Abramovich, C. D. Aliprantis Invitaion to Operator Theory. Amer. Math. Soc., Graduate Studies in Math., Vol.50, (2002).

[2] C. D. Aliprantis, O. Burkinshaw, Positive Operators, Springer, Dordrecht. (2006).

[3] C. D. Aliprantis, O. Burkinshaw, The components of a positive operator, Math. Z., 184(2) (1983), pp. 245-257. 
[4] Ben Amor M.A., M. Pliev, Laterally continuous part of an abstract Uryson operator, Int. J. of Math. Analysis, Vol.7, 58 (2013), pp. 2853-2860.

[5] A. Getoeva, M. Pliev, Domination problem for orthgonally additive operators in lattice-normed spaces, Int. J. of Math. Analysis, Vol.9, 27 (2015), pp. 1341-1352.

[6] Gumenchuk A. V., Pliev M. A., Popov M. M., Extensions of orthogonally additive operators. Mat. Stud. v. 41, no. 2, (2014). pp. 214-219.

[7] J. Flores, C. Ruiz, Domination by positive narrow operators, Positivity 7 (2003), pp. 303-321.

[8] T. JeCh, Set Theory, Springer, Berlin, 2003.

[9] A. G. Kusraev, Dominated Operators, Kluwer Acad. Publ., Dordrecht-BostonLondon (2000).

[10] A. G. Kusraev, S. S. Kutateladze, Boolean valued analysis. Springer (1999).

[11] A. G. Kusraev, M. A. Pliev, Orthogonally additive operators on lattice-normed spaces, Vladikavkaz Math. J. 3 (1999), pp. 33-43.

[12] A. G. Kusraev, M. A. Pliev, Weak integral representation of the dominated orthogonally additive operators, Vladikavkaz Math. J. 4 (1999), pp. 22-39.

[13] A. G. Kusraev, M. A. Strizhevski, Lattice-normed spaces and dominated operators, Studies on Geometry and Functional Analysis. Vol.7. Trudy Inst. Mat.(Novosibirsk), Novosibirsk, 1987, pp. 132-158.

[14] W. A. J. Luxemburg, A. C. Zannen, Riesz Spaces. Vol. 1, North Holland Publ. Comp., Amsterdam-London, 1971.

[15] O. V. Maslyuchenko, V. V. Mykhaylyuk, M. M. Popov, A lattice approach to narrow operators, Positivity 13 (2009), pp. 459-495.

[16] J. M. Mazón, S. Segura de León, Order bounded ortogonally additive operators, Rev. Roumane Math. Pures Appl. 35, 4 (1990), pp. 329-353.

[17] J. M. Mazón, S. Segura de León, Uryson operators, Rev. Roumane Math. Pures Appl. 35, 5(1990), pp. 431-449.

[18] PAGTer, DE B, The components of a positive operator, Indag. Math. 48(2) (1983), pp. 229-241.

[19] M. Pliev, Narrow operators on lattice-normed spaces, Cent. Eur. J. Math. 9, No 6 (2011), pp. 1276-1287.

[20] M. PLIEv, Uryson operators on the spaces with mixed norm, Vladikavkaz Math. J. 3 (2007), pp 47-57.

[21] M. Pliev, M. Popov, Narrow orthogonally additive operators, Positivity, v.18, 4, (2014). pp. 641-667.

[22] M. Pliev, M. Popov, Dominated Uryson operators, Int. J. of Math. Analysis, v.8, 22, (2014). pp. 1051-1059.

[23] M. Popov, B. Randrianantoanina, Narrow Operators on Function Spaces and Vector Lattices, De Gruyter Studies in Mathematics 45, De Gruyter (2013).

[24] B. Z. VulikH, Introduction to the Theory of Partially Ordered Spaces, New-York, (1967).

South Mathematical Institute of the Russian Academy of Sciences, Str. Markusa 22, Vladikavkaz, 362027 Russia

E-mail address: maratpliev@gmail.com 